$\mathrm{mt}$ and WT littermates. Electrophysiological studies in Isolated Langendorff DSG2wt/mt and WT hearts showed comparable ventricular action potential duration and effective refractory periods. DSG2 mutation correlated with increased arrhythmia inducibility after endurance training. Ventricular arrhythmias were induced in 5 of 8 DSG2wt/mt, but in none of $7 \mathrm{WT}$ hearts during right ventricular stimulation by a single extrastimulus $(p=0.03)$. In conclusion, endurance training reveals an ARVC-like phenotype in otherwise healthy and morphologically inconspicuous DSG2wt/mt mice presenting right ventricular dilatation, decreased right ventricular contractility and increased inducibility of ventricular arrhythmias during right ventricular pacing.

Abstract 111 Table 1

\begin{tabular}{lll}
\hline Genotype & WT & DSG2 mt/wt \\
\hline $\mathrm{n}$ (females/males) & $8(6 / 2)$ & $9(7 / 2)$ \\
HR (bpm) & $424 \hat{\mathrm{A}} \pm 6$ & $435 \hat{\mathrm{A}} \pm 7$ \\
RVlav d $\left(\mathrm{mm}^{2}\right)$ & $3.67 \hat{\mathrm{A}} \pm 0.20$ & $4.29 \hat{\mathrm{A}} \pm 0.28^{*}$ \\
RVsav d $\left(\mathrm{mm}^{2}\right)$ & $4.76 \hat{\mathrm{A}} \pm 0.28$ & $6.26 \hat{\mathrm{A}} \pm 0.29^{*}$ \\
RVsav s (mm & $2.10 \hat{\mathrm{A}} \pm 0.26$ & $3.50 \hat{\mathrm{A}} \pm 0.25^{*}$ \\
RV FAC (\%) & $55 \hat{\mathrm{A}} \pm 4$ & $44 \hat{\mathrm{A}} \pm 3^{*}$ \\
Age (weeks) & $21 \hat{\mathrm{A}} \pm 0.2$ & $21 \hat{\mathrm{A}} \pm 0.2$ \\
Weight (g) & $24.3 \hat{\mathrm{A}} \pm 0.7$ & $25.5 \hat{\mathrm{A}} \pm 1.6$ \\
\hline
\end{tabular}
FOLLOWING ABLATION OF THE SINUS NODE IN THE GOAT

doi:10.1136/heartjnl-2012-301877b.112

${ }^{1} \mathrm{Z}$ Borbas, ${ }^{*}{ }^{2} \mathrm{~J}$ Caldwell, ${ }^{2} \mathrm{~B}$ Prendergast, ${ }^{2} \mathrm{~A}$ Vohra, ${ }^{1} \mathrm{M}$ Boyett, ${ }^{1} \mathrm{H}$ Dobrzynski, ${ }^{2} \mathrm{C}$ Garratt. 'University of Manchester, Manchester, UK; ${ }^{2}$ Manchester Royal Infirmary, Manchester, UK

Introduction The development of sinus node dysfunction in patients may be accompanied by the emergence of subsidiary atrial pacemakers (SAPs) and/or junctional escape rhythms. It has been postulated that biological modification of SAPs may provide a potential alternative to implantation of artificial pacemakers for the treatment of sinus node disease ("biological pacemakers"). We investigated the structural and functional characteristics of SAPs in a chronic goat model.

Methods Adult female goats $(n=7)$ underwent epicardial radiofrequency catheter ablation of the sinus node, via right thoracotomy. The endpoint for ablation was defined as a fall of heart rate by $50 \%$ accompanied by change in $\mathrm{P}$ wave morphology, or the emergence of a junctional rhythm. An epicardial pacing system was implanted for continuous monitoring. Single lead surface ECG, corrected sinus node/subsidiary atrial pacemaker recovery time (CSNRT, CART) and interrogation of the implanted pacemaker were performed at baseline and weekly for 4 weeks following recovery from anaesthetic. After 4 weeks epicardial mapping was performed and location of any SAPs determined.

Results Epicardial mapping preablation confirmed sinus rhythm in all goats and guided attempts at ablation. The predefined acute endpoint of ablation was achieved in six goats. In five goats the ablated surface area was no greater than a $1 \times 2 \mathrm{~cm}$, occupying the cranial half of the intercaval region near the crista terminalis, but was more extensive in two goats (including the animal with failed acute endpoint). Despite profound postablation bradycardia, no animal required pacing for more than the first $24 \mathrm{~h}$ post-ablation. Follow-up monitoring revealed an abnormal CART (>500 ms) in three goats, and the $\mathrm{P}$ wave morphology remained different from the preablation state in five goats, consistent with a non-sinus rhythm. Repeat epicardial mapping 4 weeks post-procedure revealed stable
SAPs located in the caudal half of the intercaval region (low right atrium) in four goats. In two animals there was evidence of recovery of sinus node function and in a further goat the SAP was located within the interatrial septum or left atrium.

Conclusion Destruction of the sinus node in this experimental model resulted in the generation of chronic SAP activity in all animals. In the majority of cases SAPs were located in the low right atrial free wall (Abstract 112 figure 1) and constitute a promising stable target for electrophysiological modification in a whole animal model of sinus node disease.

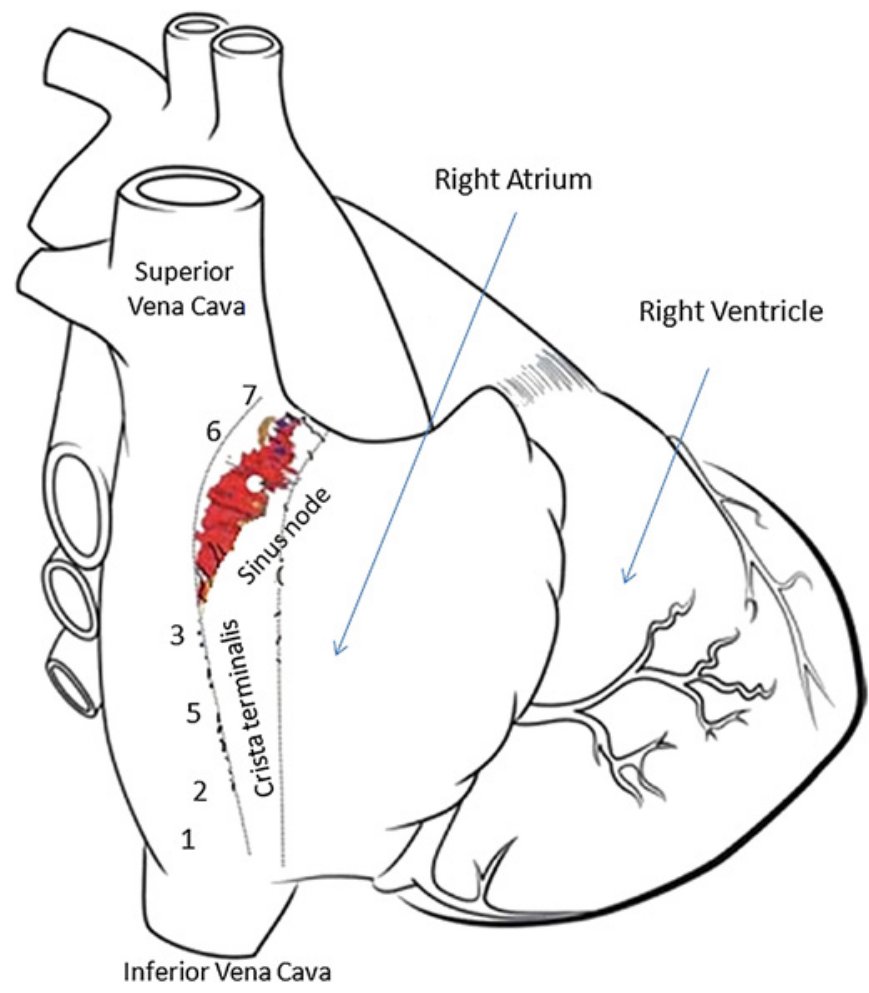

Abstract 112 Figure 1 Schematic diagram of location of subsidiary atrial pacemakers. Each number represent the location of SAP of an individual goat (1-7). Animal number 4 not displayed due to inaccessible SAP location.

\section{BLOOD THROMBOGENICITY IS INVERSELY RELATED TO CORONARY LESION SEVERITY IN PATIENTS WITH NON ST-ELEVATION ACUTE CORONARY SYNDROME AND TYPE 2 DIABETES MELLITUS}

doi:10.1136/heartjnl-2012-301877b.113

${ }^{1} \mathrm{G}$ V Viswanathan, ${ }^{1} \mathrm{~A}$ Harper, ${ }^{1}$ Karthik Balasubramaniam,* $2 \mathrm{~J} J$ Badimon, ${ }^{1} \mathrm{~S}$ M Marshall, ${ }^{1} \mathrm{~A} Z \mathrm{Z}$ Zaman. ${ }^{1}$ Newcastle University, Newcastle, UK; ${ }^{2}$ The Mount Sinai School of Medicine, New York, New York, USA

In patients with type 2 diabetes mellitus (T2DM), non-ST elevation-acute coronary syndrome (NSTE-ACS) occurs frequently secondary to non-obstructive coronary lesions than those without T2DM. Pathophysiological events leading to acute myocardial infarction in these individuals remain largely unknown, and subsequently demonstrate a poorer prognosis than those without T2DM. Methods We evaluated blood thrombogenicity (BT) in 80 patients with and without T2DM 7-10 days after troponin positive NSTEACS. In accordance to AHA/ESC recommendations all patients received secondary prevention therapy including aspirin and clopidogrel (300 mg loading and $75 \mathrm{mg}$ maintenance doses respectively). Coronary artery disease (CAD) was quantified by coronary 